\title{
ECHANTILLONNEUR BENTHIQUE D'EAUX COURANTES PEU PROFONDES UTILISANT UNE POMPE HYDRAULIQUE A MOTEUR
}

\author{
par D. ROY 1
}

Les échantillonneurs classiques utilisés en eau courante pour étudier des formes limnobiontes (faciès lénitique) ont une efficacité réduite en présence d'un substrat mixte. L'emploi d'une pompe hydraulique aspirant directement les sédiments permet de résoudre ce problème. L'auteur décrit un appareil portatif et autonome permettant 'd'échantillonner aussi bien les substrats meubles poreux que les fonds rocheux peu profonds. Un double système de filtration sépare immédiatement l'échantillon en deux classes dimensionnelles.

\section{The use of a hydraulic pump for sampling the benthos of shallow flowing water.}

Classic samplers used in flowing waters to study limnobiontic forms (lenitic aspects) have a reduced efficiency when sampling a mixed substrate. The probleme can be solved by the use of a hydraulic pump which directly aspirates the sediments. A self running portable apparatus is described which can sample not only porous movable substrates but also moderately deep rocky bottoms. A double system of filtration immediately separates the sample into two phases of different dimensions.

\section{1. - INTRODUCTION}

Les techniques d'échantillonnage couramment utilisées dans l'étude des eaux courantes peu profondes sont multiples et, pour la plupart, adaptées à un type de milieu et de synusie. Ces méthodes ont été recensées par Schwœrbel (1970), Hynes (1971), Tuffery (1971) et Elliot et Tullett (1978). On pourra se reporter également aux travaux de Macan (1958), Albrecht (1959) et Cummins (1962).

Chaque type d'appareil est limité dans son utilisation par l'hétérogénéité physique du milieu et la discontinuité observée dans la répartition des organismes. En faciès lotique, la pression du courant permet d'utiliser des échantillonneurs fixes tel que le filet de Surber. La stabilité de la masse d'eau en faciès lénitique favorise par contre l'emploi d'échantillonneurs actifs (benne, drague, écope à filet) particuliè-

1. Laboratoire d'Hydrobiologie (ERA 702 du C.N.R.S.), Université Paul-Sabatier, 118, route de Narbonne, 31077 Toulouse Cedex, France.

Adresse actuelle: Université de Montréal, Département de Biologie, C. P. 6128, Montréal 101. Québec, Canada. 
rement efficaces sur des fonds de nature sédimentaire, mais qui deviennent totalement inutilisables en présence de substrat de forte granulométrie (cailloux, pierres, blocs). De plus, les benthomètres fixes (e.g. Neill 1938 ; Sadowski 1948 ; Withley 1962), plus généralement utilisés dans ce type de milieu, sont longs et peu commodes à manipuler, et ne permettent pas de récolter aisément tous les organismes.

L'appareil que nous décrivons ici a été conçu pour étudier la dynamique des populations larvaires d'Allogamus auricollis (Pictet), trichoptère à étui arénacé abondant dans les torrents pyrénéens. Bien que cette espèce tolère des courants modérés $(25-50 \mathrm{~cm} / \mathrm{s}$ ) (Décamps 1967), surtout aux derniers stades de développement, elle occupe de préférence les zones abritées et les cuvettes rocheuses peu profondes, fréquentes en période d'étiage. Elle forme, en aval de certaines agglomérations, des populations dont la densité atteint fréquemment quelques milliers d'individus au mètre carré. L'appareil permet de prélever indistinctement les organismes qui occupent le substrat rocheux et la surface des sédiments.

\section{2. - DESCRIPTION DE L'APPAREIL}

L'appareil (fig. 1) comprend deux parties: une pompe centrifuge portative (1) reliée par un court tuyau à un cylindre de filtration (2).

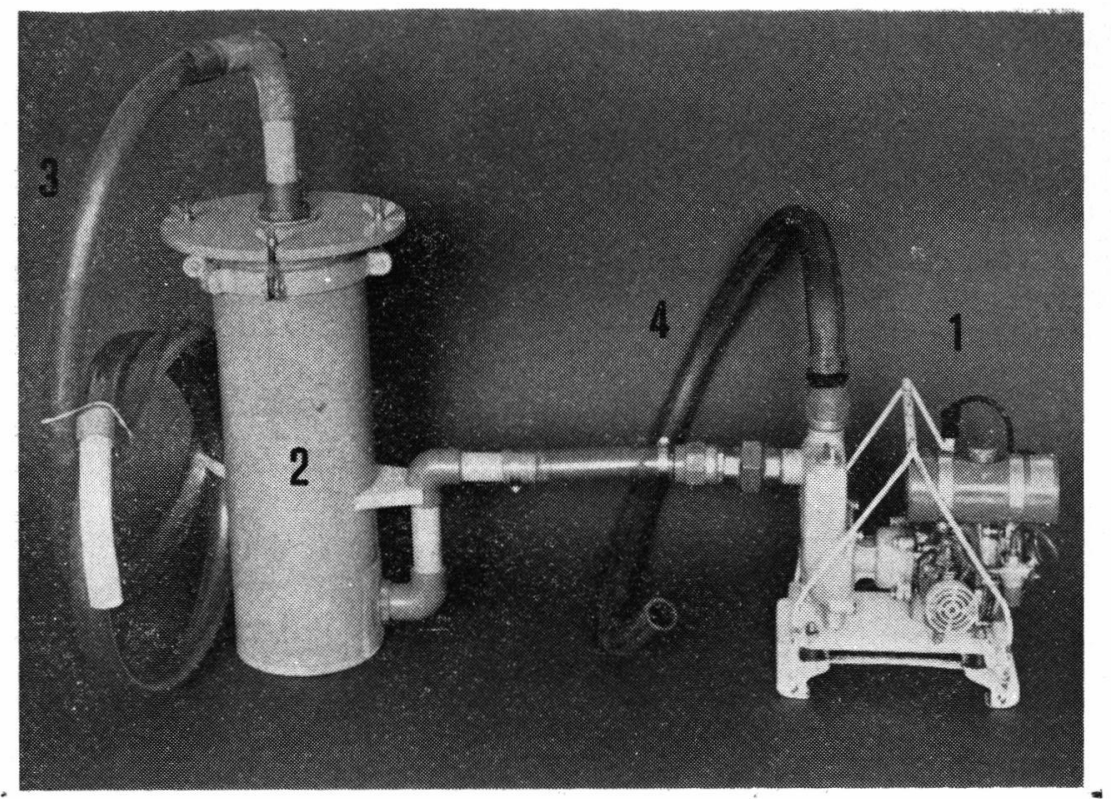

FIG. 1. - Photographie de l'appareil : (1) pompe centrifuge; (2) cylindre de filtration; (3) typau aspirateur; (4) tuyau d'évacuation de l'eau filtrée. 
L'ensemble fonctionne selon le principe simple de l'aspirateur à poussières, c'est-à-dire que l'échantillon est prélevé directement sur le fond à l'aide d'un tuyau flexible (3) et filtré à l'intérieur du cylindre avant d'être évacué (4).

La pompe centrifuge (modèle TAS PCP-3, Tanaka-Kogyo Cie Ltée, Japon) est actionnée par un moteur deux temps de $24 \mathrm{~cm}^{3}$. Sa capacité d'aspiration varie de $0,48 \mathrm{l} / \mathrm{s}$ (à bas régime) à 1,62 $\mathrm{l} / \mathrm{s}$. L'ensemble, de taille réduite, pèse $7,5 \mathrm{~kg}$.

Le cylindre de filtration (fig. 2) est un tube creux en plastique rigide (PVC) de $1 \mathrm{~cm}$ d'épaisseur, d'un diamètre interne de $15 \mathrm{~cm}$ et d'une longueur de $48 \mathrm{~cm}$. Une extrémité est obturée en permanence ; l'autre est fermée par un couvercle (5) d'un diamètre plus grand $(25 \mathrm{~cm})$ au centre duquel est vissé un court tuyau (6) (diamètre 2,5 cm), égale-

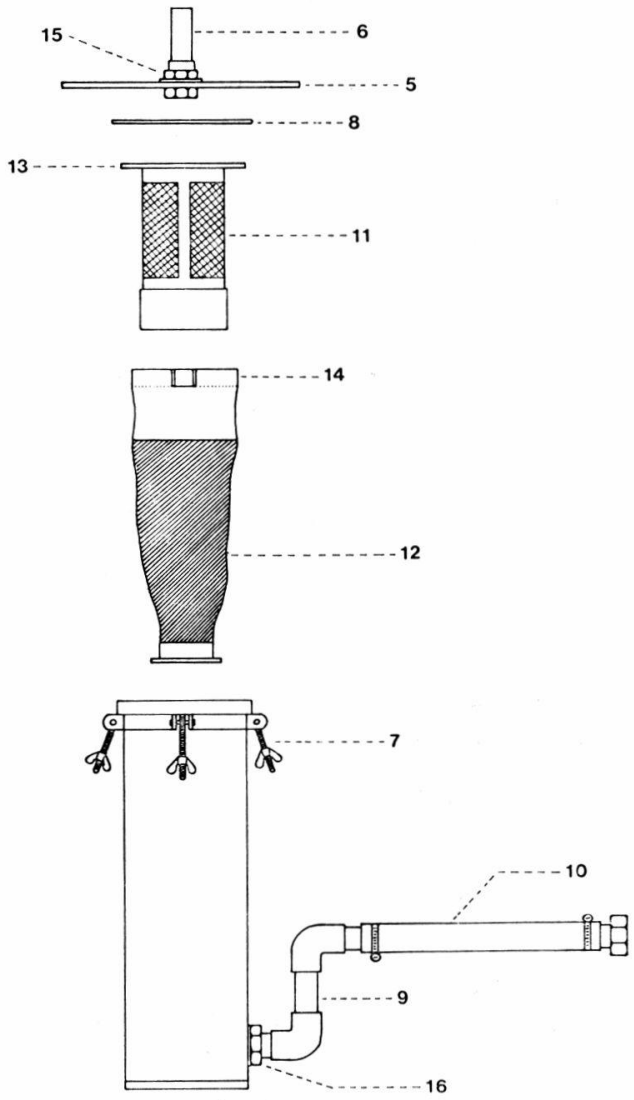

FIG. 2. - Schéma des principales composantes de l'appareil de filtration : (5) couvercle; (6) raccord du tuyau aspirateur; (7) vis à ailettes mobiles ; (8) joint circulaire en caoutchouc; (9) tuyau d'évacuation; (10) raccord de la pompe centrifuge; (11) tube grillagé; (12) filet nylon; (13) collerette de retenue ; (14) bande métallique recourbée servant de ressort; (15) et (16) boulon et joint de caoutchouc formant un raccord étanche. 
ment en PVC, où se fixe le long boyau flexible servant à l'aspiration. L'ensemble est retenu au cylindre par un système simple de 4 vis mobiles et boulons à ailettes (7) disposé en croix tout autour de l'embouchure. L'étanchéité est assurée par un joint circulaire en caoutchauc (8) de $3 \mathrm{~mm}$ d'épaisseur. L'évacuation de l'eau se fait au bas du cylindre par un tuyau coudé rigide (9) (diamètre 2,5 $\mathrm{cm}$ ) raccordé à la pompe par un tuyau transparent, souple et non compressible, terminé par un écrou (10).

Le système de filtration se compose d'un cylindre creux (11) de $10 \mathrm{~cm}$ de diamètre et long de $18 \mathrm{~cm}$, dont les parois et le fond amovible sont grillagés (vide de maille $1 \mathrm{~mm}$ ) pour retenir les sédiments grossiers, et d'un filet nylon (12) dont la maille doit être fonction de la granulométrie du fond et de la taille des organismes étudiés (ici, $270 \mu \mathrm{m})$. Le cylindre grillagé est maintenu en place grâce à une collerette (13) qui s'appuie sur un rebord interne de la paroi de l'appareil. Le filet est retenu par une bande métallique recourbée (14) formant un ressort qui le plaque contre la paroi. Pour éviter un colmatage trop rapide du filet et du grillage, on a intérêt à augmenter au maximum la surface filtrante; le filet de nylon peut ainsi occuper tout l'intérieur de l'appareil et le tube grillagé, la plus grande partie de ce filet.

La parfaite étanchéité des raccords (15) et (16) est assurée par des joints de caoutchouc facilement compressible et des écrous de préférence à des raccords plastiques collés fragiles à basse température.

\section{3. - UTILISATION SUR LE TERRAIN}

L'appareil peut être facilement transporté et utilisé par un seul manipulateur. Pendant la mise en marche du moteur, l'extrémité du tuyau aspirateur est maintenue sous l'eau par un lest. Lorsque le milieu est parfaitement statique, la parcelle de fond à échantillonner est délimitée par un simple cadre d'aluminium posé sur le substrat et la couche superficielle des sédiments est aspirée directement en déplaçant l'extrémité du tuyau aspirateur un peu au-dessus du fond, tout en écartant de la main, après les avoir soigneusement nettoyés, les cailloux ou les débris susceptibles d'obstruer l'appareil. Lorsque le milieu est turbulent, on utilise une cage quadrangulaire ou circulaire à parois hautes; on remue alors vigoureusement les sédiments tout en aspirant les matières ainsi remises en suspension. 


\section{4. - DISCUSSION}

L'usage d'une pompe hydraulique pour l'échantillonnage quantitatif des organismes aquatiques n'est pas nouveau. C'est une technique couramment utilisée pour l'étude du plancton en eaux profondes (Aron 1958, 1962 ; Omaly 1966). En océanographie, plusieurs type d'appareils fonctionnant sensiblement selon le même principe servent à prélever le macro- et le meiobenthos des sédiments meubles et rocheux (Brett 1974 ; Massé 1967, 1970 ; Emig et Lienhart 1967; Gulliksen et Deras 1975) ; ceux-ci exigent toutefois la présence d'un plongeur en scaphandre autonome pour diriger l'aspiration. En eaux peu profondes, Heurteaux et Marazanof (1965) utilisent une pompe aspirante manuelle et une enceinte de capture pour étudier le telmoplancton et le telmobenthos (à l'exclusion des formes macrobenthiques) des marais de Camargue.

L'appareil a d'abord été conçu pour l'échantillonnage épibenthique en eaux profondes et utilisé, de la surface, dans un lac d'altitude des Pyrénées (Dupin et Giani 1972) ; aucun prélèvement quantitatif n'a cependant pu être obtenu de cette façon. Adapté aux eaux courantes peu profondes (inférieures à $1 \mathrm{~m}$ ), celui-ci s'est révélé particulièrement efficace. En présence de sédiments meubles poreux, il est possible d'échantillonner $0,10 \mathrm{~m}^{2}$ de la couche superficielle sans colmatage du filet ou du grillage; dans le cas d'un substrat rocheux, la surface d'échantillonnage peut atteindre $0,25 \mathrm{~m}^{2}$.

Cet appareil permet de récolter la totalité des organismes benthiques présents (à l'exclusion du microbenthos de taille inférieure au vide de maille du filet); il est parfaitement quantitatif. L'utilisation du double système de filtration a l'avantage de séparer automatiquement le prélèvement en deux classes de taille. Son efficacité est toutefois limitée par la profondeur et la vitesse du courant; l'utilisateur doit être en mesure de voir et d'atteindre aisément le lit du cours d'eau. Il devient pratiquement inutilisable en présence d'une forte concentration de sédiments meubles compacts (limons et argiles) et à des températures de l'air inférieures à $0{ }^{\circ} \mathrm{C}$. On peut lui reprocher également d'être un peu plus encombrant que les appareils traditionnels et d'abîmer dans une proportion plus élevée les organismes les plus frêles, tels certains Plécoptères et Ephéméroptères.

\section{TRAVAUX CITES}

Albrecht (M. L.). 1959. - Die quantitative Untersuchung der Bodenfauna fliessender Gewasser (Untersuchungsmethoden und Arbeitsergebnisse). Z. Fisch. N $F ., 8: 481-550$. 
ARON (W.). 1958. - The use of a large capacity portable pump for plankton sampling with notes on plankton patchiness. J. Mar. Res., 16: 158-173.

ARoN (W.). 1962. - Some aspects of sampling the macroplankton. Cons. Perm. Int. Explor. Mer, Rapp. Proc. Verb., 153 : 29-38.

BRETT (C. E.). 1964. - A portable hydraulic diver-operated dredge-sieve for sampling subtidal macrofauna. J. Mar. Res., 22 : 205-222.

Cummins (K.W.). 1962. - An evaluation of some techniques for the collection and analysis of benthic samples with special emphasis on lotic waters. Amer. Midl. Nat., 67 : 477-504.

Décamps (H.). 1967. - Ecologie des trichoptères de la vallée d'Aure (Hautes-Pyrénées). Annls Limnol., 3 : 399-577.

Dupin (B.) et Giani (N.). 1972. - Etude des populations benthiques d'un lac de hautes montagnes: le lac de Port-Bielh $(2285 \mathrm{~m})$ (Hautes-Pyrénées). Thèse $3^{\text {c }}$ cycle, Toulouse.

Elliot (J. M.) et TUlletT (P.A.). 1978. - A bibliography of samplers for benthic invertebrates. Freshwat. Biol., Occ. Publ., 4 : 1-61.

Emig (C.) et Lienhart (R.). 1967. - Un nouveau moyen de récolte pour les substrats meubles infralitoraux: l'aspirateur sous-marin. Rec. Trav. St. Mar. End., 42 : 115-120.

Gulliksen (B.) et Deras (K. M.). 1975. - A diver-operated suction sampler for fauna on rocky bottoms. Oikos, $26: 246-249$.

Heurteaux (P.) et Marazanof (F.). 1965. - Une méthode de prélèvement quantitatif en écologie aquatique. Annls Limnol., 1 : 191-196.

Hynes (H. B. N.). 1971. - Benthos of flowing water. In Edmonson (W. T.) et WiNBERG (G. G.). A manual on methods for the assessment of secondary productivity in fresh waters. I.B.P. Handbook, $\mathrm{n}^{\circ} 17$, Oxford, Blackwell, $358 \mathrm{p}$.

Macan (T. T.). 1958. - Methods of sampling the bottom fauna in stony streams. Mitt. int. Verein. Limnol., $8: 1-21$.

MASSE (H.). 1967. - Emploi d'une suceuse hydraulique transformée pour les prélèvements dans les substrats meubles infralitoraux. Helgolander wiss. Meeresunters., $15: 500-505$.

Masse (H.). 1970. - La suceuse hydraulique, bilan de quatre années d'emploi, sa manipulation, ses avantages et ses inconvénients. Peuplements benthiques. Tethys, 2 : 547-556.

NeILL (R. M.). 1938. - The food and feeding of the brown strout (Salmo trutta L.) in relation to the organic environment. Trans. $R$. Soc. Edinb., $59: 481-520$.

Omaly (N.). 1966. - Moyens de prélèvements du zooplancton. Essai historique et critique. Pélagos, $5: 1-169$.

SADowsKi (A. A.). 1948. - Benthomètre. Nouvel appareil pour les prélèvements quantitatifs de zoobenthos dans les rivières de montagne (en russe). Soobchtch. A. A. Grouz. S.S.R., 9: 365-368.

SchWoerbel (J.). 1970. - Methods of hydrobiology (Freshwater biology). Oxford, Pergamon Press, $200 \mathrm{p}$.

TUFFery (G.). 1971. - L'échantillonnage des peuplements d'inventébrés des eaux continentales courantes. In Lamotre (M.) et Bourlière (F.). Problèmes d'écologie: l'échantillonnage des peuplements animaux des milieux aquatiques. Paris, Masson et $\mathrm{C}^{\mathrm{ie}}$, éd., $294 \mathrm{p}$.

Whitley (L. S.). 1962. - New sampler for use in shallow streams. Limnol. Ocean., $7: 265-6$. 